\title{
THE AEROSPACE PERFORMANCE FACTOR: UTILIZATION OF THE ANALYTICAL HIERARCHY PROCESS TO DEVELOP A BALANCED PERFORMANCE AND SAFETY INDICATOR OF THE NATIONAL AIRSPACE SYSTEM FOR THE FEDERAL AVIATION ADMINISTRATION
}

\author{
Thomas Michael Lintner \\ Office of Safety \\ Federal Aviation Administration \\ Washington, D.C., USA \\ E-mail: thomas.lintner@faa.gov \\ Steven D. Smith \\ Office of Safety \\ Federal Aviation Administration \\ Washington, D.C., USA \\ E-mail: steven.d.smith@faa.gov \\ Scott Smurthwaite, $\mathrm{PhD} *$ \\ Office of Safety \\ Federal Aviation Administration \\ Washington, D.C., USA \\ E-mail: scott.smurthwaite@faa.gov
}

\begin{abstract}
The central mission of the Federal Aviation Administration (FAA) is the safety of the national airspace system (NAS). Historically, basic metrics such as traffic counts, delays, flight cancellations, incidents, and accidents were used to gauge performance. This method of analysis was effective at looking at isolated events but failed to take into account an overall system-wide assessment of the national airspace system. Currently, the Office of Safety is developing the Aerospace Performance Factor (APF) in order to better understand the overall system-wide events and their relative contribution to the overall safety of the national airspace. In this process, the APF utilizes aspects of the Analytical Hierarchy Process (AHP) method to establish structure and create a measurement of historical FAA aerospace incidents. It presents the results of the measurement as a graphical representation of the system's performance over time. The APF is an effective tool for assessing the relative performance of the FAA's airspace and has been adopted by other groups within the aviation arena including the European-based commercial airline easyJet, the Irish Aviation Authority (IAA), the United Kingdom's National Air Traffic Services (NATS), and the European Organisation for the Safety of Air Navigation (EUROCONTROL).
\end{abstract}

Keywords: Aerospace Performance Factor, Analytical Hierarchy Process, national airspace system, Office of Safety, Federal Aviation Administration

* Corresponding author 


\section{Traditional methods of assessing performance}

Generally, air navigation service providers (ANSP's) have relied on isolated metrics to assess the performance of their systems. Information such as annual traffic counts and annual flight delays have traditionally been used as a method of quantifying and reporting on the system's performance. These reports might state that in 2008 Airport-X supported x-amount of flights and had y-amount of total delays, resulting in z-amount of total minutes of delays. Then when compared to 2007, the results for the same airport might show a $13 \%$ increase in overall traffic with a $10 \%$ increase in delays for an overall increase of $z$-amount of minutes in delays. Although these reports were effective the overall efficiency and general performance trend was unclear.

Other views of reporting performance were also effective for individual lines of business but were still less able to view system-wide impact. A sample report might state that in 2008 there were $\mathrm{x}$-amount of mishaps and when compared to 2007 there was a $3 \%$ increase while traffic increased by $12 \%$. These snapshot reports, which have traditionally been used by the FAA and other international ANSP's, provide a quick view of important portions of air traffic control systems but do not fully take into account other factors that contribute to a better system-wide view of the overall program.

Despite the narrow scope of the reporting, individual incident or mishap reports contain substantial details. For example, a report of a specific event might contain extensive details on the circumstances of the incident. A severity rating associated with assessing the risk of a mishap would be generated using vector mathematics. Similar details were gathered on other incident types. If a truck was inadvertently driven onto a runway, a runway incursion (RI) incident report would be filed and as part of the details of the incident a rating of $\mathrm{A}, \mathrm{B}, \mathrm{C}$, or D would be assigned to the report with a classification of $\mathrm{A}$ being most significant and $\mathrm{D}$ being the least significant. These individual reports on the performance aspects of the air traffic control (ATC) system were effective for specific aspects of the system but were not aggregated into a comprehensive report that would highlight a system-wide view of the performance of the NAS.

\section{The need for multiple safety indicators}

In order to better assess the overall system-wide performance, it was recognized that all relevant safety reports would need to be integrated into a single unified measurement. By including all reports (including subject matter expert's assessments) all data could be included in the measurement. By following this approach, where all data are placed 'on the table' for all to see, a comprehensive multi-factor indicator could be developed which might identify current performance, long-term trends, and ultimately allow for the better management of the overall system.

Recognizing there would be numerous issues associated with creating new reporting criteria, and knowing the importance of historical data to support trending, a decision was made to utilize all of the traditional safety reports. The spectrum of traditional data collected, and used within the APF, included the following:

- Operational Errors (OE), where less than the prescribed separation exists between two aircraft. (OE's are subdivided as Category A, B, C, D, PE, MVA, and Flight Formations, depending on certain criteria.)

- Operational Deviations (OD), where an aircraft infringed on an area of airspace.

- Pilot Deviations (PD), where the pilot deviated from a rule or ATC instruction.

- Runway Incursions (RI), where there is an unauthorized aircraft, vehicle, or person on a runway. (RI's are subdivided as Category A, B, C, and D depending on certain criteria.)

- Near Midair Collisions (NMAC) where a pilot has reported an encounter with another aircraft that the pilot considered potentially unsafe. 
- Vehicle and Pedestrian Deviations (VPD), a type of RI involving the unauthorized presence of a vehicle or person on a runway.

By capitalizing on existing safety reports which spanned various areas of ATC operations it was believed the total impact that each of these indicators had on the total performance of the system could be established. What was less clear was how these specific reports would be included into a comprehensive measure. For example, what was the effect of a NMAC and a PD? Similarly, what was the relative contribution of the RI with rating of $\mathrm{C}$ to the overall performance of the system? What impact do PD's have on the overall assessment of the performance and safety of the national airspace system?

\section{The Analytic Hierarchy Process}

\subsection{Background of AHP}

The Analytic Hierarchy Process (AHP) is based on the experience gained by its developer, T.L. Saaty, while directing research projects in the US Arms Control and Disarmament Agency. It was developed as a reaction to the finding that there is a lack of common, easily understood, and easy-to-implement methodology to enable the making of complex decisions (Bhushan and Ria, 2004). Since its development, the simplicity and power of the AHP has lead to its rapid and widespread use across multiple domains beyond the process of making complex decisions.

\subsection{The AHP process}

Given its simplicity and ease of use, and relying on the three primary functions of AHP (structuring complexity, measurement, and synthesis), decision-maker's thoughts on the problem can be organized in a manner that is simple to follow and analyze. These characteristics make AHP an effective methodology to a wide range of applications and allow any complex situation that requires structuring, measurement, and synthesis to be better understood.

In addition to the three primary functions, there are three related basic principles of AHP (decomposition, comparative judgments, and hierarchical composition or synthesis of priorities) which are utilized in the AHP methodology. The decomposition principle is applied to structure a complex problem into a hierarchy of clusters and sub-clusters. The principle of comparative judgments is applied to construct pairwise comparisons of all combinations of elements in a cluster with respect to the parent of the cluster. These pairwise comparisons are used to derive 'local' priorities of the elements in a cluster with respect to their parent. The principle of hierarchical composition or synthesis is applied to multiply the local priorities of the elements in a cluster by the 'global' priority of the parent element, producing global priorities throughout the hierarchy and then adding the global priorities for the lowest level elements.

\subsection{Steps in AHP}

Saaty (2008) describes the steps in the AHP in the following way:

1. Define the problem and determine the kind of knowledge sought.

2. Structure the decision hierarchy from the top with the goal of the decision, then the objectives from a broad perspective, through the intermediate levels (criteria on which subsequent elements depend) to the lowest level (which usually is a set of the alternatives).

3. Construct a set of pairwise comparison matrices. Each element in an upper level is used to compare the elements in the level immediately below with respect to it.

4. Use the priorities obtained from the comparisons to weigh the priorities in the level immediately below. Do this for every element. Then for each element in the level below add its weighted 
values and obtain its overall or global priority. Continue this process of weighting and adding until the final priorities of the alternatives in the bottom most level are obtained.

In this process users of the AHP decompose their problem into a hierarchy of more easily comprehended sub-problems, each of which can be analyzed independently. Once the hierarchy is built, subject matter experts systematically evaluate the elements by comparing them to one another two at a time. The AHP then converts these evaluations to numerical values that can be processed and compared over the entire range of the problem. From this process, a numerical weight is derived for each element of the hierarchy.

\section{The Aerospace Performance Factor}

The APF does not use the AHP as a multi-criteria decision tool. Since no decision is being sought, no alternatives are used in the APF AHP. Utilizing the AHP the APF looked at all available contributing factors which could be used to measure the overall safety performance of the system. All available reported factors that involved safety issues were included to ensure a complete system-wide view.

\subsection{Building the hierarchy}

All factors were obtained through the use of historical mishap reports of events and incidents within the system. The elements chosen were comprised of Operational Errors (OE), Pilot Deviations (PD), Near Midair Collisions (NMAC), Operational Deviations (OD), Runway Incursions (RI), and Vehicle and Pedestrian Deviations (VPD). The elements were then placed in a hierarchy, using a mindmap, that represents the logical relationships of the elements to each other. The completed structures of the elements are displayed in Figure 1. 


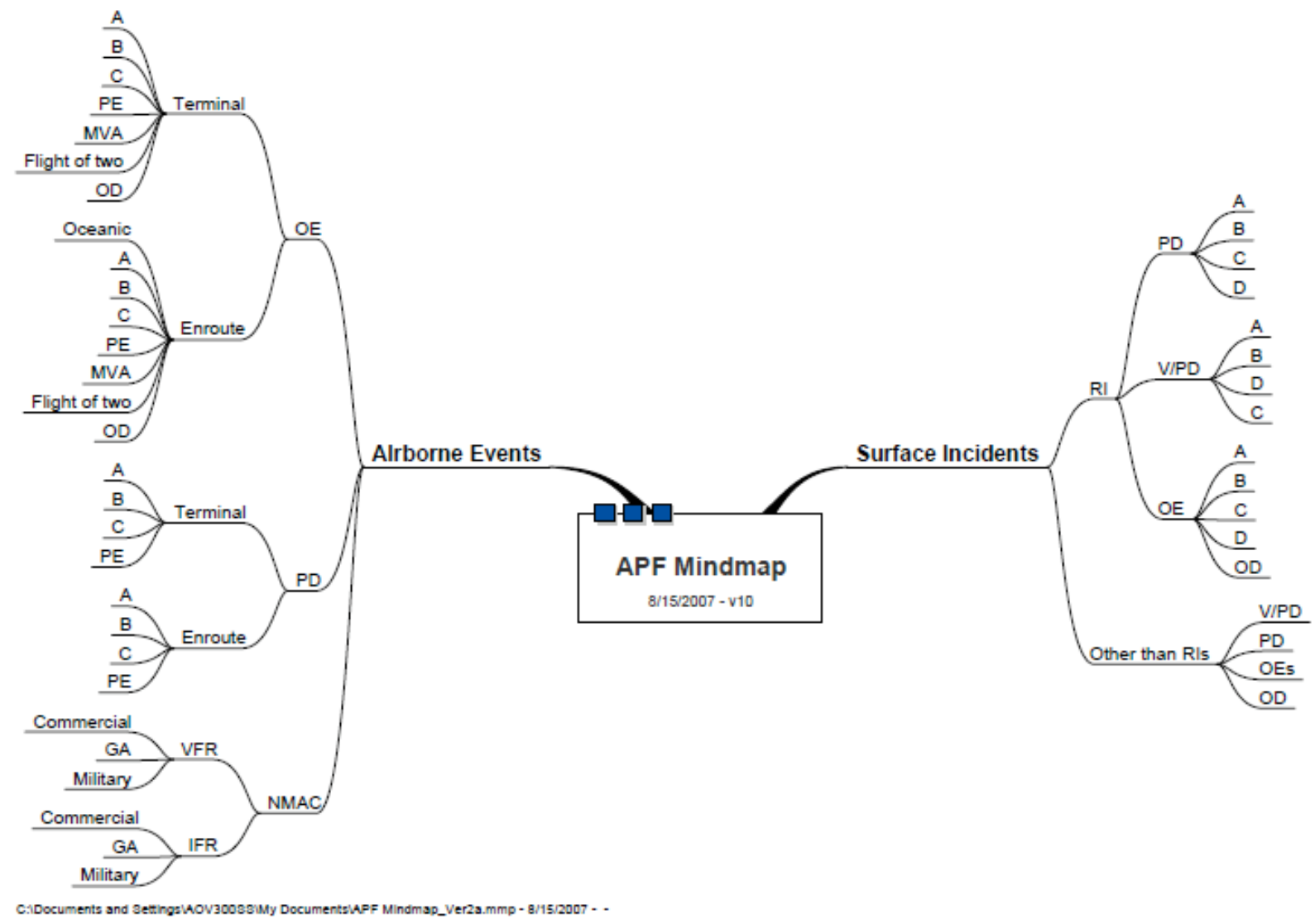

Figure 1 . The national airspace hierarchy structure.

\subsection{Judging the pairwise comparisons}

Once the hierarchy was built, subject matter experts systematically evaluated the various elements by comparing them to one another two at a time (Table 1). The subject matter experts were presented with the paired comparison and a rating scale (Table 2) and asked "Between these 2 choices, how strongly do you feel that this item represents a risk to the safety of the system? By making the comparison of the various elements the experts used their judgments about the elements' relative significance and a ratio scale was created (Table 3 ).

Table 1. Sample Airborne events pairwise comparison matrix.

\begin{tabular}{|c|c|c|c|}
\hline & $\begin{array}{l}\text { Operational Errors } \\
(\mathrm{OE})\end{array}$ & $\begin{array}{l}\text { Pilot Deviations } \\
\text { (PD) }\end{array}$ & $\begin{array}{l}\text { Near Midair Collision } \\
\text { (NMAC) }\end{array}$ \\
\hline $\begin{array}{l}\text { Operational Errors } \\
\text { (OE) }\end{array}$ & |-------------------------- & & \\
\hline $\begin{array}{l}\text { Pilot Deviations } \\
\text { (PD) }\end{array}$ & --------------------------- & --------------------------- & \\
\hline $\begin{array}{l}\text { Near Midair Collision } \\
\text { (NMAC) }\end{array}$ & ---------------------------- & ------------------------- & ----------------------------- \\
\hline
\end{tabular}


Table 2. Rating scale.

\begin{tabular}{|c|c|c|c|c|c|c|c|c|}
\hline Equal & $\begin{array}{l}\text { Between } \\
\text { Equal and } \\
\text { Moderately }\end{array}$ & Moderately & $\begin{array}{c}\text { Between } \\
\text { Moderately } \\
\text { and } \\
\text { Strongly }\end{array}$ & Strongly & $\begin{array}{c}\text { Between } \\
\text { Strongly } \\
\text { and } \\
\text { Very } \\
\text { Strongly }\end{array}$ & $\begin{array}{c}\text { Very } \\
\text { Strongly }\end{array}$ & $\begin{array}{c}\text { Between } \\
\text { Very } \\
\text { Strongly } \\
\text { and } \\
\text { Extreme }\end{array}$ & Extreme \\
\hline
\end{tabular}

Table 3. Sample Airborne events pairwise comparison matrix [note: these are sample data].

\begin{tabular}{|l|l|l|l|}
\hline & $\begin{array}{l}\text { Operational Errors } \\
(\mathrm{OE})\end{array}$ & $\begin{array}{l}\text { Pilot Deviations } \\
(\mathrm{PD})\end{array}$ & $\begin{array}{l}\text { Near Midair Collision } \\
\text { (NMAC) }\end{array}$ \\
\hline $\begin{array}{l}\text { Operational Errors } \\
(\mathrm{OE})\end{array}$ & ------------------------- & $1 / 5$ & $1 / 2$ \\
\hline $\begin{array}{l}\text { Pilot Deviations } \\
(\mathrm{PD})\end{array}$ & -------------------------- & ----------------------- & 5 \\
\hline $\begin{array}{l}\text { Near Midair Collision } \\
(\mathrm{NMAC})\end{array}$ & --------------------------- & ------------------------- & -------------------------- \\
\hline
\end{tabular}

In this example of Airborne events (Table 3) a judgment was made that an Operational Error represents a greater risk than a Pilot Deviation and that Pilot Deviation represented a greater risk than a Near Midair Collision.

\subsection{Deriving the weighted score from the matrix}

The AHP then converts these pairwise evaluations created by the subject matter experts and the following weights are created (Table 4).

Table 4. Sample weighted score for Airborne events [note: these are sample data].

\begin{tabular}{|l|c|}
\hline & Weighted Score \\
\hline Operational Errors (OE) & 0.65 \\
\hline Pilot Deviations (PD) & 0.20 \\
\hline Near Midair Collision (NMAC) & 0.15 \\
\hline TOTAL & 1.00 \\
\hline
\end{tabular}

\subsection{Calculating the element score}

Once the judgment evaluations are converted to numerical values the data is applied to each of the branches of the model for a particular time period. For any element the total count would be divided by the total count for traffic for that particular element and then multiplied by its calculated weight.

$\mathrm{X}=($ incident count $\mathrm{x}$ weight $) /$ traffic count

\subsection{Summing the element scores}

The same calculations were then performed on each element of the hierarchy to calculate each element's relative contribution to the overall model. By calculating each element of the branches comprising a collection of the hierarchy and summing the score, the relative contribution of the element and branch to 
the overall model could be 'rolled-up' and overall scores could be determined. In so doing, all safety elements are 'rolled up' to create a cumulative value on the overall system.

\subsection{Summing the sums}

In the same way that individual elements within an incident-type were 'rolled-up' to give a branch its relative score; branches were 'rolled-up' and relative scores were attached to them. By summing up the calculated values of the branches of the hierarchy and continuing this 'roll-up' process until the root of the hierarchy is reached a comprehensive system-wide performance measurement tool was complete.

Total score for an event $=\Sigma$ (sub-events)

\section{Putting it all together}

The goal of the APF was to create a visual representation of the performance of the national airspace system. To achieve this, the final step in this process was to apply real data based on the existing historical safety indicators.

\subsection{Applying the model to real data}

By defining the elements of the model (and having subject matter experts quantify the relative contribution of the elements in the model) the APF tool could be applied to series of real data. A comparison of the model for a specified period of time would reveal the relative contribution of each of the elements and branches of the model.

\subsection{Performance over time}

The relative contribution of the elements (and branches) of the model to the performance of the airspace could also be applied to time-series data collected over many years. By examining the performance of the system over time a view of the historical performance could lead to an expectation of the future direction (Figure 2). 
All

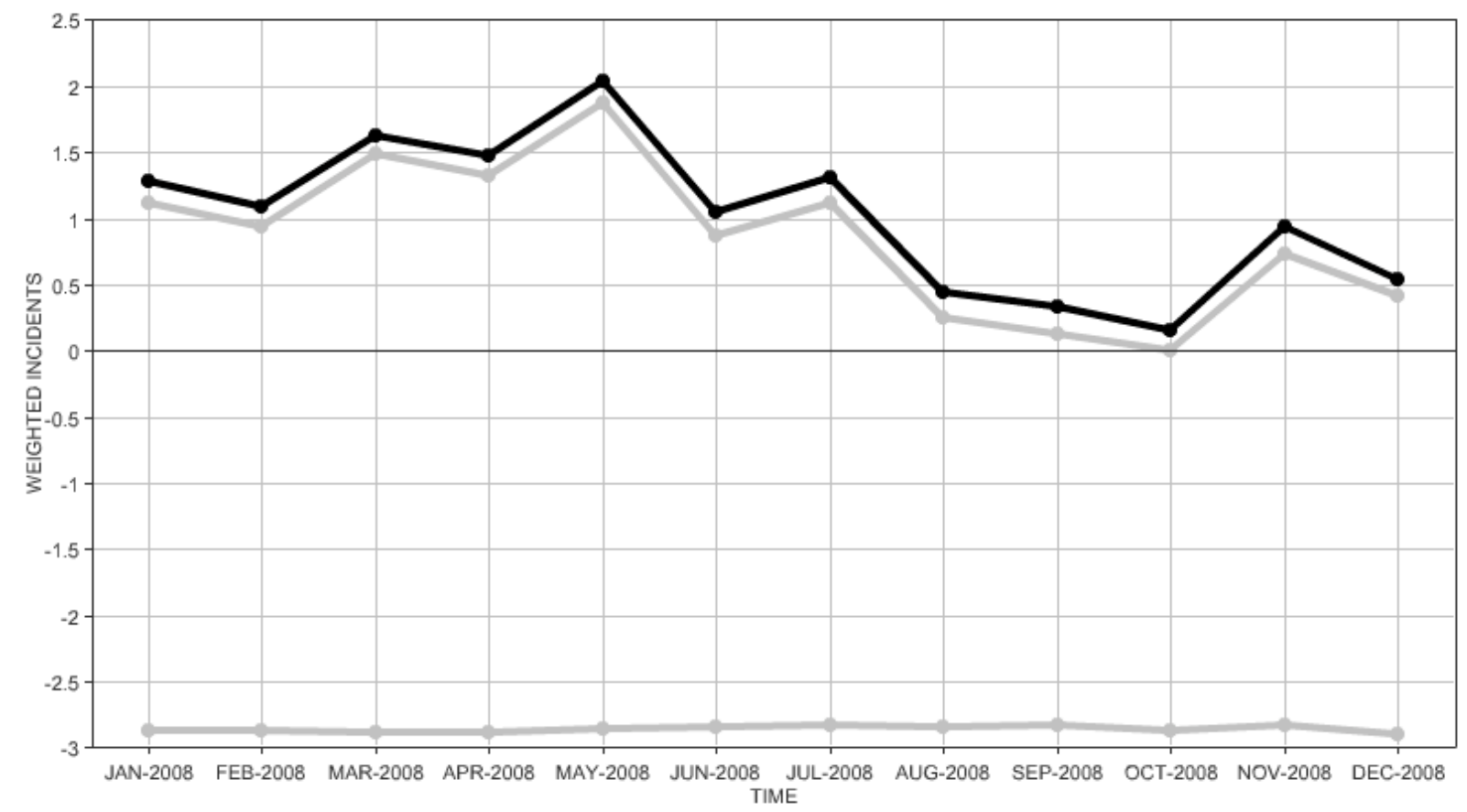

Figure 2. System-wide view of performance of the national airspace system.

Figure 2 shows the performance of the national airspace during 2008. The dark line represents the total performance of the national airspace and the lightly shaded lines represent the sub-categories of the hierarchy (Airborne and Surface). The lightly shaded line directly below the dark top line represents the contribution of Airborne incidents while the lightly shaded line nearer the $\mathrm{x}$-axis represents the relative contribution of Surface incidents. By summing these two sub-categories the total is shown in black.

\subsection{Establishing a baseline}

The performance of the national airspace analyzed across time allowed for a direct comparison to other time periods. For example, the performance of the system could be compared to an agreed upon baseline. The baseline in Figure 2 is indicated by the horizontal line at the zero point of the y-axis. The baseline is calculated by taking the mean of the 36 monthly values of the performance for the years 2004 and 2006 . This time period was selected because it represented real data over an extended period. By establishing this baseline a visual comparison can be made between a particular point in time and the baseline. 


\subsection{An interactive APF}

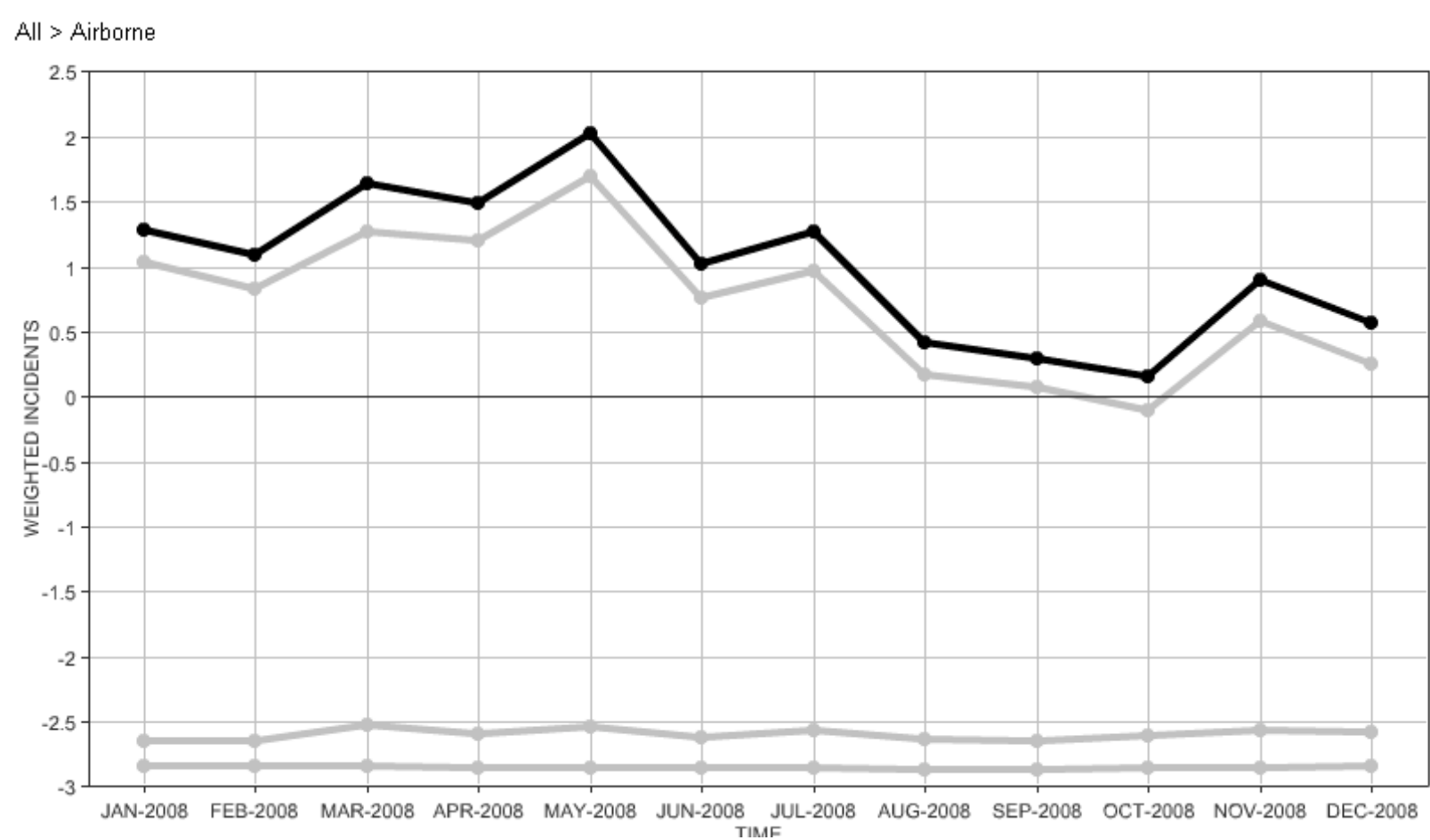

Figure 3. An interactive APF.

In order for the APF to be interactive the figures allow the user to click on the sub-category lines of the figure and display the underlying data associated with this sub-category. For example, Figure 2 displays the two sub-categories that comprise the black line (i.e., performance of the system). By clicking on the sub-category lines (in this example the Airborne line) a new figure is displayed with the details for this sub-category (Figure 3).

When the user navigates to a new figure all the functionalities of the figure remain the same but are calculated for the chosen category. For example, the baseline is calculated for the Airborne incidents calculated by taking the mean of the 36 monthly Airborne values for the years 2004 and 2006. The zero point on the y-axis then represents the mean value for Airborne on dates between Jan 2004 to Dec 2006. By establishing this baseline the comparisons of any given point in time is always being measured against an established baseline particular to that category.

In addition to displaying a baseline particular to the user-chosen category, the APF application also displays the sub-categories that comprised that category (Figure 3). In the example of Airborne incidents, the categories making up Airborne are displayed. The top grey line is the Pilot Deviations (PD), the middle grey line is the Operational Errors (OE), and the bottom line is the Near Midair Collision (NMAC). In the same manner that the other lines on the figures allow the user to 'drill-down' the lines on Figure 3 also allow the user to make a selection and viewing the details of the sub-categories that comprise the category (Figure 4). 


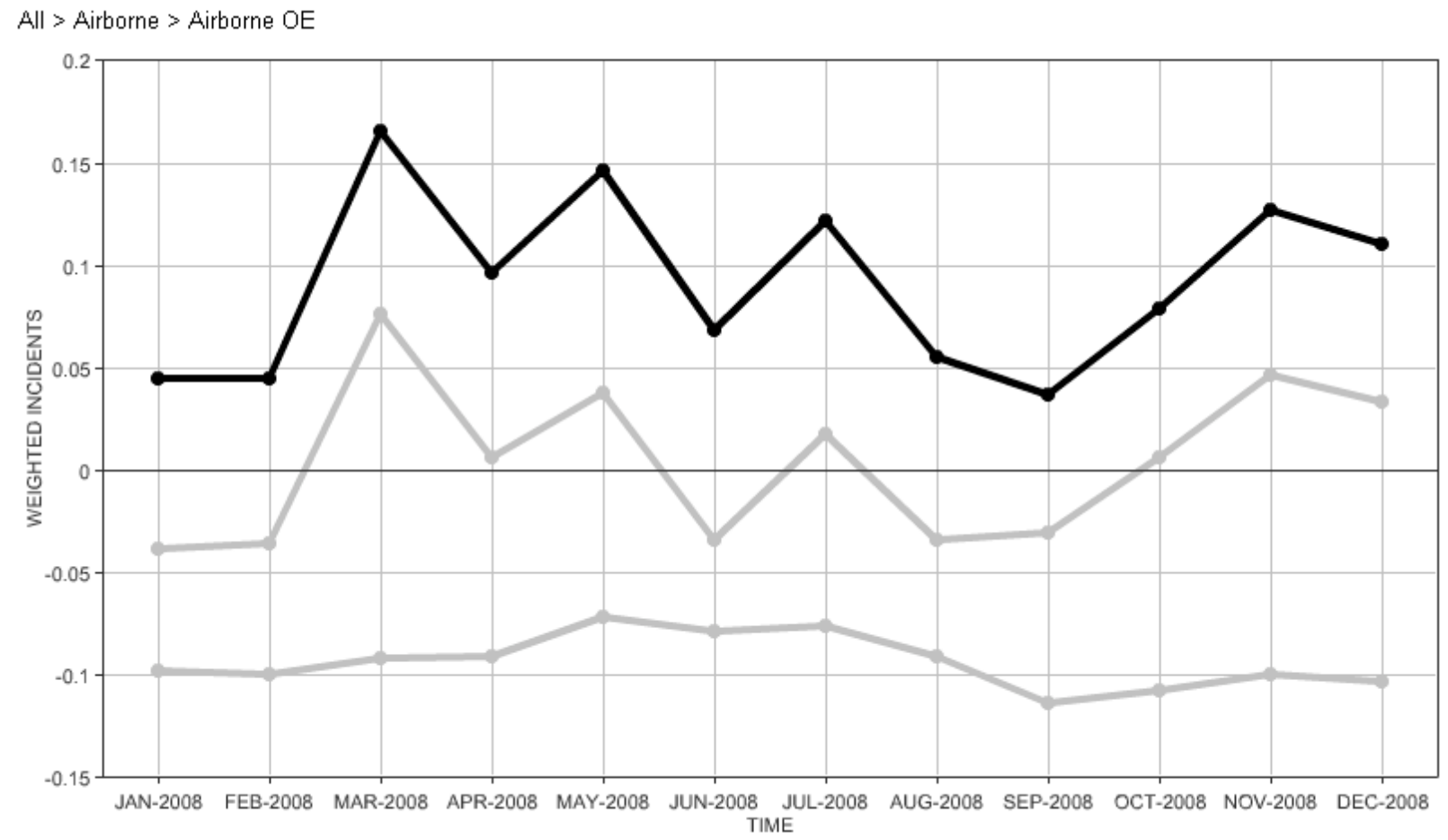

Figure 4. An interactive APF: ALL > Airborne > Airborne OE.

\section{Conclusion}

By adhering to the three primary functions (structuring complexity, measurement, and synthesis) and applying three related basic principles of AHP (decomposition, comparative judgments, and hierarchical composition or synthesis of priorities) a visual representation of the performance of the system over time was created.

The APF was created by defining the elements of the model and having subject matter experts judge the relative importance of the elements in the model. Once this was completed the APF tool was applied to a series of real data and a picture of the performance of the entire landscape of the national airspace emerged.

Based on the success of this effort other groups within the arena of aviation are working to develop their own APF's. Recently the European-based commercial airline easyJet created an APF based on their industry-specific categorization structure and data. In addition to easyJet, who is also being supported by the Imperial College of London, several European ANSP's are assessing the incorporation of APF methodology into their operations.

This paper describes the process of establishing an indicator of performance within the arena of aviation, however, given the flexibility of the AHP framework, the process of providing a visual representation of performance can be applied to other industries. 


\section{REFERENCES}

Bhushan, N. and Ria, K. (2004). Strategic Decision Making: Applying the Analytic Hierarchy Process, London: Springer-Verlag London Limited.

Saaty, T.L. (2008). Decision making with the analytic hierarchy process. Int., J. Services Sciences, 1(1), 83-98. 